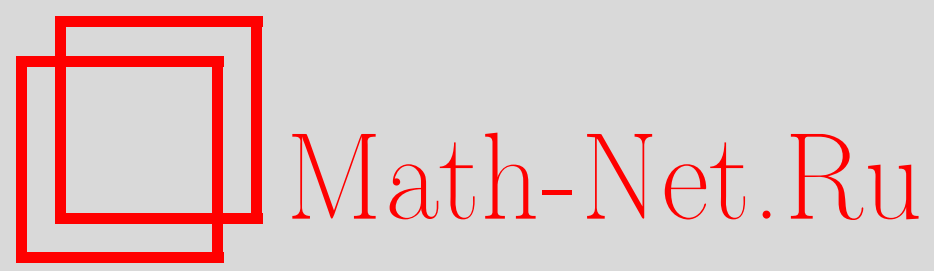

Н. Н. Хрисанов, Интегральный метод аппроксимации экспериментальных данных экспоненциальными функциями, Вестн. Сам. гос. техн. ун-та. Сер. Физ.мат. науки, 2001, выпуск 12, 195-199

DOI: https://doi.org/10.14498/vsgtu84

Использование Общероссийского математического портала Math-Net.Ru подразумевает, что вы прочитали и согласны с пользовательским соглашением

http: //www. mathnet.ru/rus/agreement

Параметры загрузки:

IP: 18.234 .197 .8

26 апреля 2023 г., 13:33:13 


\section{Н.Н. Хрисанов}

\section{ИНТЕГРАЛЬНЫЙ МЕТОД АППРОКСИМАЦИИ ЭКСПЕРИМЕНТАЛЬНЫХ ДАННЫХ ЭКСПОНЕНЦИАЛЬНЫМИ ФУНКЦИЯМИ}

Описывается метод аппроксимации экспериментальных данных в виде линейной комбинации экспоненциальных функций по интегральным характеристикам от исследуемого процесса, позволяющий повысить точность определения параметров экспоненциальной модели в условиях помех.

Для аппроксимации выборочных данных, полученных, например, при исследовании переходных процессов, часто используют линейную комбинацию экпоненциальных функций (экспонент):

$$
h(t) \approx \sum_{i=1}^{n} A_{i} \exp \left(-\lambda_{i} t\right),
$$

где $A_{i}$ - вещественные числа; $\lambda_{i}$ - могут быть как вещественными (апериодический процесс), так и комплексными (колебательный процесс).

Применение метода наименьших квадратов определения параметров экспоненциальной модели ( $A_{i}$ и $\left.\lambda_{i}\right)$ в данном случае приводит к очень сложной нелинейной задаче оптимизации. Свести задачу к решению линейных уравнений позволяет метод Прони [1]. Однако исходный метод Прони очень чувствителен к ошибкам в экспериментальных данных и не позволяет использовать избыточные экспериментальные данные для повышения точности. Использовать избыточные данные позволяет так называемый обобщенный метод Прони, в котором на первом и третьем этапах применяется метод наименьших квадратов. Однако эксперименты показали, что зависимость от погрешностей в экспериментальных данных остается высокой. В данной работе предлагается определять параметры экспоненциальной модели по интегральным характеристикам переходных процессов.

Как известно [2], функция (1) обладает тем свойством, что существует линейная зависимость между ее $(n+1)$ равноотстоящими ординатами

$$
\sum_{i=0}^{n} c_{i} h(t+i T)=0
$$

где $c_{i}$ - постоянные числа; $T$ - интервал между отсчетами.

Показатели $\lambda_{i}$ могут быть получены подстановкой (1) в (2) и решением алгебраического уравнения

$$
c_{0}+\sum_{i=1}^{n} c_{i} \xi^{i}=0
$$

Уравнение (3) имеет $n$ корней $\xi_{i}, i=\overline{1, n}$. Показатели $\lambda_{i}$ определяются по формуле

$$
\lambda_{i}=-\frac{\ln \xi_{i}}{T} .
$$

Найдем определенные интегралы от (1) на следующих участках: $\left[t_{0}, \infty\right],\left[t_{0}+T, \infty\right]$, $\left[t_{0}+2 T, \infty\right], \ldots,\left[t_{0}+n T, \infty\right]$, где $t_{0}$ - момент времени, соответствующий первому отсчету; $T$ фиксированный интервал времени. Отметим, что $\exp (-\lambda t)=0$ при $t=\infty$. Для $i$-того участка интеграл $H_{i}$ будет равен

$$
H_{i}=-\sum_{i=1}^{n+1} \frac{A_{i}}{\lambda_{i}} \exp \left\{\left[-\lambda_{i}\left[t_{0}+(i-1) T\right]\right\} .\right.
$$

Составим уравнение, связывающее линейной зависимостью $H_{i}$ :

$$
\sum_{i=0}^{n} c_{i} H_{i+1}=0 .
$$

Подставив (5) в (6), получим:

$$
\sum_{i=1}^{n} \frac{A_{i}}{\lambda_{i}} \exp \left(-\lambda_{i} t_{0}\right)\left[c_{0}+\sum_{k=1}^{n} c_{k} \exp \left(-\lambda_{i} k T\right)\right]=0 .
$$


После подстановки $\xi_{i}=\exp \left(-\lambda_{i} T\right)$, получим :

$$
\sum_{i=1}^{n} \frac{A_{i}}{\lambda_{i}} \exp \left(-\lambda_{i} t_{0}\right)\left[c_{0}+\sum_{k=1}^{n} c_{j} \xi_{i}^{k}\right]=0 .
$$

Выражение в левой части (7) равно нулю при выполнении $c_{0}+\sum_{k=1}^{n} c_{j} \xi_{i}{ }^{k}$, что аналогично уравнению (3). Таким образом, показатели могут быть определены по значениям интегралов (5) от экспериментальной зависимости, путем решения алгебраического уравнения (3) и подстановкой его корней в (4). Заметим, что значение $t_{0}$ не входит в формулы для определения показателей, Это важно в случае, когда трудно определить момент времени, соответствующий первому отсчету.

Поскольку число неизвестных в (1) равно $2 n$, то, для определения коэффициентов $c_{i}$ необходимо решить следующую систему линейных уравнений (коэффициент $c_{n}$ нормируется к единице):

$$
\left\{\begin{array}{llllll}
c_{0} H_{1}+c_{1} H_{2} & \ldots & +c_{n-1} H_{n} & +H_{n+1} & =0, \\
c_{0} H_{2}+c_{1} H_{3} & \ldots & +c_{n-1} H_{n+1} & + & H_{n+2} & =0, \\
& \cdot & & \cdot & \cdot & \\
c_{0} H_{n}+c_{1} H_{n+1} & \ldots & +c_{n-1} H_{2 n-1} & +H_{2 n} & =0 .
\end{array}\right.
$$

Амплитуды $A_{i}$ могут быть определены из $n$ значений $H_{1}, H_{2}, \ldots, H_{n}$ определенных интегралов от $h(t)$, полученных интегрированием экспериментальной зависимости, например, на интервалах $\left[t_{0}, \infty\right],\left[t_{0}+2 T, \infty\right],\left[t_{0}+4 T, \infty\right], \ldots,\left[t_{0}+(n-1) T, \infty\right]$ и решением следующей системы уравнений:

$$
\left\{\begin{array}{llll}
A_{1}^{\prime} & +A_{2}^{\prime} & \ldots+A_{n}^{\prime} & =H_{1}, \\
A_{1}^{\prime} p_{1} & +A_{2}^{\prime} p_{2} & \ldots+A_{n}^{\prime} p_{n} & =H_{2}, \\
& \cdot & & \cdot \\
A_{1}^{\prime} p_{1}^{n-1}+A_{2}^{\prime} p_{2}^{n-1} & \ldots+A_{1}^{\prime} p_{n}^{n-1}=H_{n},
\end{array}\right.
$$

где $A_{i}^{\prime}=A_{i} \exp \left(-\lambda_{i} t_{0}\right) / \lambda_{i}, \quad p_{i}=\exp \left(-\lambda_{i} T\right), i=\overline{1, n}$. Определив из (9) $n$ значений $A_{i}^{\prime}$, получаем окончательно

$$
A_{i}=A_{i} \lambda_{i} .
$$

Как было указано выше, определение вещественных показателей экспонент производится в соответствии с (4). В том случае, когда уравнение (3) имеет комплексно-сопряженные корни $\xi_{k, k+1}=x \pm j y$, показатели экспонент (которые в этом случае также будут комплексносопряженными) определяются следующим образом:

$$
\lambda_{k, k+1}=-\frac{1}{T}[\ln z \pm j(\theta+2 \pi v)],
$$

где $z=\sqrt{x^{2}+y^{2}}, \theta=\operatorname{arctg}(y / x), v$ - целое число и нуль.

При подстановке в аппроксимирующую функцию (1) пары сопряженных показателей, соответствующие им слагаемые можно заменить выражениями:

$$
A_{k} \exp (c t) \cos (d t) \text { и } A_{k+1} \exp (c t) \sin (d t),
$$

где $c=\ln z / T, d=\theta / T$.

Взяв определенные интегралы от этих выражений в соответствующих пределах, получим формулы для соответствующих коэффициентов системы линейных уравнений (9):

$$
\begin{aligned}
& a_{k, i}=p_{k 0} p_{k}^{i-1}\left[-c \cos \left(t^{*}\right)+d \sin \left(t^{*}\right)\right], \\
& a_{k+1, i}=p_{k 0} p_{k}^{i-1}\left[d \cos \left(t^{*}\right)-c \sin \left(t^{*}\right)\right],
\end{aligned}
$$

где $p_{k 0}=\exp \left(c t_{0}\right) / c^{2}+d^{2}, p_{k}=\exp (2 c T), t^{*}=d\left[t_{0}+2 T(i-1)\right]$.

Произведем оценку погрешности определения $A_{i}$ и $\lambda_{i}$ с помощью описанного выше метода. Пусть дан набор экспериментальных данных, измеренных в моменты времени $t_{i}=t_{0}+i \Delta t$, 
$i=0,1, \ldots$ с систематической погрешностью $\Delta$, а средне-квадратичное отклонение (с.к.о.) случайной погрешности равно $\sigma$.

Предельные погрешности $\gamma_{\lambda_{i}}$ и $\gamma_{A_{i}}$ определения $\lambda_{i}$ и $A_{i}$ могут быть вычислены по формуле:

$$
\gamma_{A_{i}}=\bar{\Delta}_{A_{i}}+t_{d} \bar{\sigma}_{A_{i}}, \quad \gamma_{\lambda_{i}}=\bar{\Delta}_{\lambda_{i}}+t_{d} \bar{\sigma}_{\lambda_{i}}
$$

где $\bar{\Delta}_{A_{i}}, \bar{\Delta}_{\lambda_{i}}$ - систематическая погрешность определения $A_{i}$ и $\lambda_{i} ; \bar{\sigma}_{A_{i}}, \bar{\sigma}_{\lambda_{i}}$ - с.к.о. случайной погрешности определения $A_{i}$ и $\lambda_{i} ; t_{d}$ - доверительный коэффициент [3].

Поскольку искомые параметры являются функциями от $H_{j}, j=\overline{1,2 n}$, воспользуемся формулами для погрешности косвенных измерений [3]:

$$
\begin{gathered}
\bar{\Delta}_{A_{i}}=\sum_{j=1}^{2 n} \frac{\partial A_{i}}{\partial H_{j}} \Delta_{j}, \quad \bar{\sigma}_{A_{i}}^{2}=\sum_{j=1}^{2 n}\left(\frac{\partial A_{i}}{\partial H_{j}}\right)^{2} \sigma_{j}^{2}+2 \sum_{k<j} \frac{\partial A_{i}}{\partial H_{k}} \frac{\partial A_{i}}{\partial H_{j}} K_{k j}, \\
\bar{\Delta}_{\lambda_{i}}=\sum_{j=1}^{2 n} \frac{\partial \lambda_{i}}{\partial H_{j}} \Delta_{j}, \quad \bar{\sigma}_{\lambda_{i}}^{2}=\sum_{j=1}^{2 n}\left(\frac{\partial \lambda_{i}}{\partial H_{j}}\right)^{2} \sigma_{j}^{2}+2 \sum_{k<j} \frac{\partial \lambda_{i}}{\partial H_{k}} \frac{\partial \lambda_{i}}{\partial H_{j}} K_{k j},
\end{gathered}
$$

где $\Delta_{j}$ - систематическая погрешность определения интеграла $H_{j} ; \sigma_{j}$ - с.к.о. случайной погрешности определения интеграла $H_{j} ; K_{k j}$ - корреляционный момент величин $H_{k}$ и $H_{j}$.

Систематическая погрешность $\Delta_{j}$ может быть вычислена по формуле:

$$
\Delta_{j}=\Delta\left[T_{\text {пр }}-(j-1) T\right],
$$

где $T_{\text {ПР }}$ - длительность исследуемого процесса.

С.к.о. $\sigma_{j}$ случайной погрешности определения $H_{j}$ при использовании методов дискретного интегрирования (например, метода трапеций) и при условии, что корреляционная функция погрешности спадает до нуля за время интервала дискретизации, равно [4]:

$$
\sigma_{j}=\sqrt{n_{j}} \Delta t \sigma,
$$

где $n_{j}$ - количество отсчетов, взятых при вычислении $H_{j} ; \Delta t$ - интервал дискретизации.

В качестве примера, оценим погрешность вычисления параметров аппроксимирующей функции, состоящей из двух экспонент:

$$
h(t) \approx A_{1} \exp \left(-\lambda_{1} t\right)+A_{2} \exp \left(-\lambda_{2} t\right) \text {. }
$$

Поскольку число неизвестных равно четырем, необходимо произвести интегрирование на четырех участках:

$$
H_{1}=\int_{0}^{\infty} h(t) d t, \quad H_{2}=\int_{T}^{\infty} h(t) d t, \quad H_{3}=\int_{2 T}^{\infty} h(t) d t, \quad H_{4}=\int_{3 T}^{\infty} h(t) d t .
$$

Система уравнений (8) примет вид:

$$
\left\{\begin{array}{l}
c_{0} H_{1}+c_{1} H_{2}+H_{3}=0 \\
c_{0} H_{2}+c_{1} H_{3}+H_{4}=0
\end{array}\right.
$$

Откуда имеем

$$
c_{0}=\frac{H_{2} H_{4}-H_{3}^{2}}{H_{1} H_{3}-H_{2}^{2}}, \quad c_{1}=\frac{H_{2} H_{3}-H_{1} H_{4}}{H_{1} H_{3}-H_{2}^{2}} .
$$

Алгебраическое уравнение (3) примет вид:

$$
c_{0}+c_{1} \xi+c_{1} \xi^{2}=0,
$$

корни которого равны:

$$
\xi_{1,2}=-\frac{c_{1}}{2} \pm \sqrt{\frac{c_{1}}{4}-c_{0}},
$$

откуда для действительных $\xi_{1,2}$ получаем:

$$
\lambda_{1,2}=-\frac{\ln \xi_{1,2}}{T} .
$$

Система уравнений (9) в данном случае будет иметь вид: 


$$
\left\{\begin{aligned}
A_{1}^{\prime}+A_{2}^{\prime} & =H_{1} \\
A_{1}^{\prime} p_{1}+A_{2}^{\prime} p_{2} & =H_{3}
\end{aligned}\right.
$$

где $p_{1}=\exp \left(-2 \lambda_{1} T\right), p_{2}=\exp \left(-2 \lambda_{2} T\right), A_{1}^{\prime}=A_{1} / \lambda_{1}, A_{2}^{\prime}=A_{2} / \lambda_{1}$.

Решив эту систему уравнений относительно $A_{1}^{\prime}$ и $A_{2}^{\prime}$, определим $A_{1}$ и $A_{2}$ :

$$
A_{1}=\frac{H_{1} p_{2}-H_{3}}{p_{2}-p_{1}} \lambda_{1}, \quad A_{2}=\frac{H_{3}-H_{1} p_{1}}{p_{2}-p_{1}} \lambda_{2} .
$$

Если $h(0)=0$, то предыдущие выражения упрощаются и принимают вид

$$
A_{1}=-A_{2}=A=\frac{H_{1} \lambda_{1} \lambda_{2}}{\lambda_{2}-\lambda_{1}}
$$

Для вычисления погрешности определения $\lambda_{1}, \lambda_{2}$ частные производные, входящие в (13), будут равны (для случая действительных корней):

$$
\frac{\partial \lambda_{i}}{\partial H_{j}}=\frac{\frac{\partial c_{1}}{2 \partial H_{j}}-\frac{1}{2 \sqrt{c_{1}^{2} / 4-c_{0}}}\left(\frac{\partial c_{1}}{2 \partial H_{j}}-\frac{\partial c_{0}}{2 \partial H_{j}}\right)}{\xi_{i} T},
$$

где $i=1,2, j=\overline{1,4}, \frac{\partial c_{0}}{\partial H_{1}}=-\frac{c_{0} H_{3}}{H_{1} H_{3}-H_{2}^{2}}, \frac{\partial c_{0}}{\partial H_{2}}=\frac{H_{1} H_{3} H_{4}+H_{2}^{2} H_{4}-2 H_{2} H_{3}^{2}}{\left(H_{1} H_{3}-H_{2}^{2}\right)^{2}}$,

$$
\begin{gathered}
\frac{\partial c_{0}}{\partial H_{3}}=\frac{2 H_{2}^{2} H_{3}-H_{1} H_{3}^{2}-H_{1} H_{2} H_{4}}{\left(H_{1} H_{3}-H_{2}^{2}\right)^{2}}, \frac{\partial c_{0}}{\partial H_{4}}=\frac{H_{2}}{H_{1} H_{3}-H_{2}^{2}}, \frac{\partial c_{1}}{\partial H_{1}}=\frac{c_{0} H_{2}}{H_{1} H_{3}-H_{2}^{2}}, \\
\frac{\partial c_{1}}{\partial H_{2}}=\frac{H_{1} H_{3}^{2}+H_{2}^{2} H_{3}-2 H_{1} H_{2} H_{4}}{\left(H_{1} H_{3}-H_{2}^{2}\right)^{2}}, \frac{\partial c_{1}}{\partial H_{3}}=\frac{H_{1}^{2} H_{4}-H_{2}^{3}}{\left(H_{1} H_{3}-H_{2}^{2}\right)^{2}}, \frac{\partial c_{1}}{\partial H_{4}}=-\frac{H_{1}}{H_{1} H_{3}-H_{2}^{2}} .
\end{gathered}
$$

Аналогично вычислим производные для $A_{1}$ и $A_{2}$ :

$$
\begin{gathered}
\frac{\partial A_{1}}{\partial H_{i}}=\frac{\left(\xi_{2}-H_{1} \frac{\partial \xi_{2}}{\partial H_{i}}\right)\left(\xi_{2}-\xi_{1}\right)-\left(H_{1} \xi_{2}-H_{3}\right)\left(\frac{\partial \xi_{2}}{\partial H_{i}}-\frac{\partial \xi_{1}}{\partial H_{i}}\right)}{\left(\xi_{2}-\xi_{1}\right)^{2}} \lambda_{2}-\frac{\left(H_{1} \xi_{2}-H_{3}\right)}{\xi_{2}-\xi_{1}} \frac{\partial \lambda_{1}}{\partial H_{i}}, \\
\frac{\partial A_{2}}{\partial H_{i}}=\frac{\left(\xi_{2}-H_{1} \frac{\partial \xi_{1}}{\partial H_{i}}\right)\left(\xi_{2}-\xi_{1}\right)-\left(H_{3}-H_{1} \xi_{1}\right)\left(\frac{\partial \xi_{2}}{\partial H_{i}}-\frac{\partial \xi_{1}}{\partial H_{i}}\right)_{\lambda_{2}}-\frac{\left(H_{3}-H_{1} \xi_{2}\right)}{\xi_{2}-\xi_{1}} \frac{\partial \lambda_{2}}{\partial H_{i}},}{\left(\xi_{2}-\xi_{1}\right)^{2}}
\end{gathered}
$$

где $i=1,3, \frac{\partial \xi_{1,2}}{\partial H_{i}}=-\frac{1}{2} \frac{\partial c_{1}}{\partial H_{i}} \pm \frac{1}{2 \sqrt{c_{1}^{2} / 4-c_{0}}}\left(\frac{c_{1}}{2} \frac{\partial c_{1}}{\partial H_{i}}-\frac{\partial c_{0}}{\partial H_{i}}\right)$.

Для случая $A_{1}=-A_{2}=A$, имеем:

$$
\begin{gathered}
\frac{\partial A}{\partial H_{1}}=\frac{\left[\lambda_{1} \lambda_{2}+H_{1}\left(\lambda_{1} \frac{\partial \lambda_{2}}{\partial H_{1}}+\lambda_{2} \frac{\partial \lambda_{1}}{\partial H_{1}}\right)\right]\left(\lambda_{2}-\lambda_{1}\right)-H_{1} \lambda_{1} \lambda_{2}\left(\frac{\partial \lambda_{2}}{\partial H_{1}}-\frac{\partial \lambda_{1}}{\partial H_{1}}\right)}{\left(\lambda_{2}-\lambda_{1}\right)^{2}}, \\
\frac{\partial A}{\partial H_{i}}=\frac{H_{1}\left[\left(\lambda_{2}-\lambda_{1}\right)\left(\lambda_{1} \frac{\partial \lambda_{2}}{\partial H_{i}}+\lambda_{1} \frac{\partial \lambda_{1}}{\partial H_{i}}\right)-\lambda_{1} \lambda_{2}\left(\frac{\partial \lambda_{2}}{\partial H_{i}}-\frac{\partial \lambda_{1}}{\partial H_{i}}\right)\right]}{\left(\lambda_{2}-\lambda_{1}\right)^{2}}, i=2,4 .
\end{gathered}
$$

Для случая, когда $A_{1}$ и $A_{2}$ : имеют разные знаки (переходной процесс имеет ярко выраженный максимум в некоторый момент времени $t_{m}$ ), на рис. 1 приведена зависимость погрешности определения показателей и амплитуды экспоненциальной модели в зависимости от интервала интегрирования, значения которого берутся относительно значения $t_{m}$ при $\Delta=0, \sigma=0,3 \%$. Как следует из графика, в качестве интервала интегрирования $T$ целесообразно выбирать значения, близкие к $t_{m}$. 


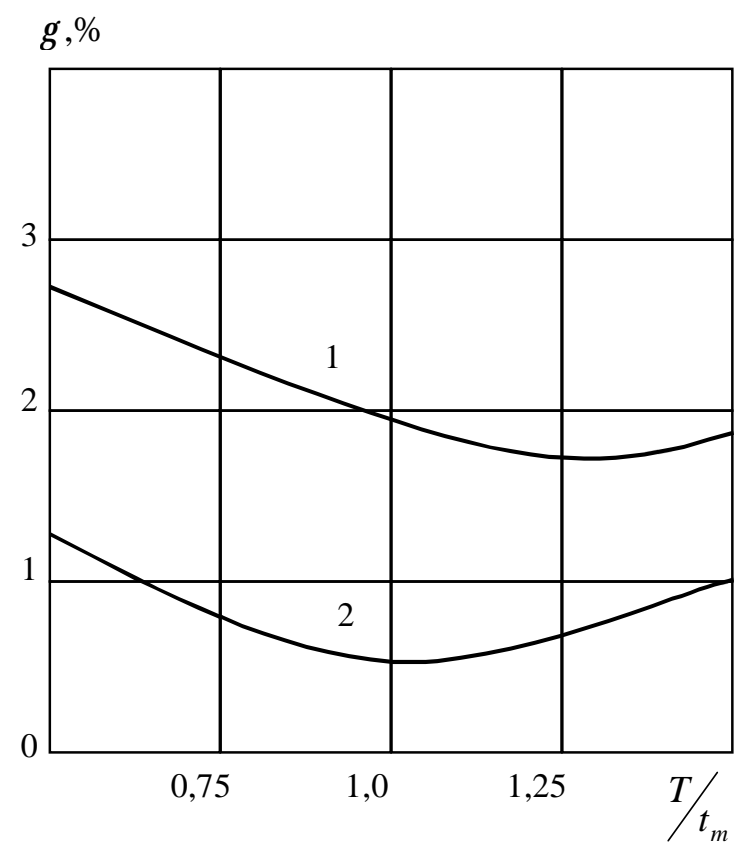

Р и с. 1. Зависимость погрешности определения параметров в зависимости от выбранного интервала интегрирования:

1-погрешность определения амплитуды;

2-погрешность определения показателей

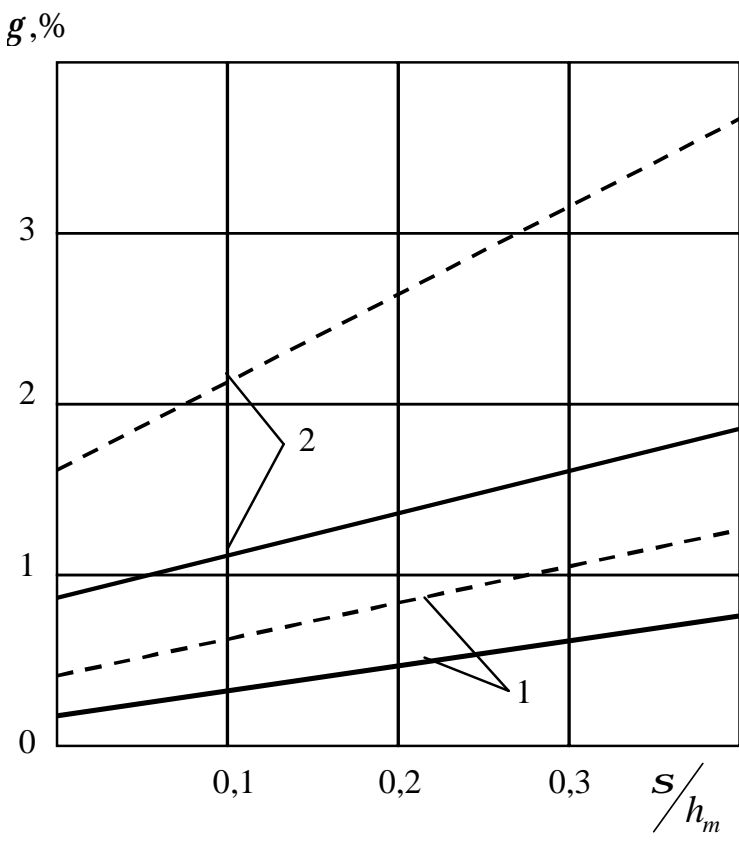

Р и с. 2. Зависимость погрешности определения параметров экспоненциальной модели в зависимости о с.к.о. случайной погрешности измерений:

сплошная линия - интегральный метод; пунктирная - метод Прони; 1 - погрешность определения амплитуды; 2- погрешность определения показателей

На рис. 2 приведены зависимости предельной погрешности определения параметров экспоненциальной модели от с.к.о. случайной погрешности измерения, при использовании интегрального метода и модифицированного метода Прони, полученные на основе статистического моделирования для модели с двумя экспонентами. Из графиков следует, что в данном случае, интегральный метод позволяет повысить точность аппроксимации экспериментальных данных экспоненциальными функциями.

\section{БИБЛИОГРАФИЧЕСКИЙ СПИСОК}

1. Марпл.-мл. С.Л. Цифровой спектральный анализ и его приложения: Пер. с англ. М.: Мир, 1990. 584 с.

2. Ланцош К. Практические методы прикладного анализа. М.: ГИФМЛ, 1961. 524 с.

3. Новиикий П.В., Зограф И.А. Оценка погрешностей результатов измерений. Л.: Энергоатомиздат, 1985. $248 \mathrm{c}$.

4. Иикович Э.Л. Контроль производства с помощью вычислительных машин. М.: Энергия, 1975. 416 с. 